\title{
Unstable processes in magnetic cataclysmic variables
}

\section{Case of the long-period polar QQ Vulpeculae}

\author{
A. V. Halevin ${ }^{1,4}$, N. M. Shakhovskoy ${ }^{2,5}$, I. L. Andronov ${ }^{1,4}$, and S. V. Kolesnikov ${ }^{3,4}$ \\ 1 Department of Astronomy, Odessa National University, T. G. Shevchenko park, 65014 Odessa, Ukraine \\ e-mail: halevin@astronomy.org.ua \\ 2 Crimean Astrophysical Observatory, Crimea, Ukraine \\ e-mail: shakh@crao.crimea.ua \\ 3 Astronomical Observatory, Odessa National University, T. G. Shevchenko park, 65014 Odessa, Ukraine \\ e-mail: il-a@mail.od.ua, astro@paco.odessa.ua \\ ${ }^{4}$ Isaac Newton Institute of Chile, Odessa Branch, Ukraine \\ 5 Isaac Newton Institute of Chile, Crimean Branch, Ukraine
}

Received 12 June 2001 / Accepted 21 June 2002

\begin{abstract}
We report the results of an investigation of unstable processes in the magnetic cataclysmic variable QQ Vul based on observations obtained at the 2.6-m Shain telescope and 1.25-m AZT-11 of the Crimean Astrophysical Observatory. The detected range of the short time-scale variations is likely caused by a sequence of different small flares, due to a non-coherence of this type variability. We report that there is practically no quasi-periodic variability in this system. Also, we have detected variations of the shot noise decay time during the orbital period. Investigations of the shot noise decay time allow us to estimate the physical size of the accreted blobs. Using archive data and our minima timings, the photometric period of this system is updated $\left(P_{\text {phot }}=0.15452040(2)\right)$.
\end{abstract}

Key words. accretion, accretion disks - instabilities - stars: individual: QQ Vul - stars: magnetic fields novae, cataclysmic variables

\section{Introduction}

AM Her type objects are close binary systems consisting of a red dwarf filling its Roche lobe, and of a strongly magnetized white dwarf with surface fields in the range of dozens to hundreds of MG (Meggitt \& Wickramasinghe 1984). In AM Her objects, or "polars", the magnetic field of the white dwarf is sufficiently strong to prevent the formation of an accretion disk, which is present in non-magnetic or weakly-magnetic cataclysmic variables. In these systems, the gas motion is determined by the balance between the magnetic pressure and the ram plus thermal pressure (Mukai 1988). When the magnetic pressure becomes larger than the ram plus thermal pressure, matter within the ballistic stream begins to be coupled to the magnetic field such that it will fall down the field lines onto the white dwarf surface. If we assume dipolar geometry for the magnetic field, then the active regions on the white dwarf surface lies near the magnetic poles. In a ballistic stream, the flow is split according to the Rayleigh-Taylor instability into

Send offprint requests to: A. V. Halevin, e-mail: halevin@astronomy.org.ua large diamagnetic blobs with sizes of about $10^{9} \mathrm{~cm}$ (Hameury et al. 1986; Beardmore \& Osborne 1997). Accretion of the most massive blobs can produce flares in such systems.

QQ Vul is a polar with a typical strength of the magnetic field of $\sim 30$ MG (Cropper 1998). QQ Vul was discovered as a polar by Nousek et al. (1984). They derived an orbital period of $\sim 222 \mathrm{~min}$. The long-term variability has been investigated by Andronov \& Yavorskij (1983), who found that it switches between high and low brightness states. Andronov \& Fuhrmann (1987) determined brightness minima of QQ Vul from photographic observations obtained in Sonneberg, Moscow and Abastumani observatories since 1928. Mukai et al. (1986) and McCarthy \& Bowyer (1986) discovered two emission regions in this system: a high speed gas in the accretion flow and the heated face of the secondary star. Osborne et al. (1986) introduced soft X-ray observations, which show the presence of two brightness minima over an orbital period. Mukai et al. (1986) determined the physical properties of the components, and interpreted the primary dip in the light curves as a combination of a self-eclipse of the accretion column and the cyclotron beaming effect. Mukai (1988) determined the geometry of the system, where they suggest, that the secondary minima are caused by the partial eclipse of the active region by the limb of the 
white dwarf, because it corresponds to the peak of linear polarization. In the second half of the 1985 year, the soft Xray curve of QQ Vul changed radically (Osborne et al. 1987). Instead of two neighboring minima, the new curve shows two unequal minima, separated by 0.5 in phase. Using the ROSAT and Ginga observations of QQ Vul, Beardmore et al. (1995) distinguished hard and soft X-ray emission regions in the system and suggest the presence of a two-pole accretion. Recently Cropper (1998) discussed week-to-week changes of the light curve. Halevin et al. (1997) investigated changes of the photometric amplitude of this star during the 1986-1988 period. Precise measurements of system parameters have been made by Catalan et al. (1999) and Schwope et al. (2000).

\section{High speed photometry and circular polarimetry of QQ Vul}

Photometric and polarimetric observations of QQ Vul have been obtained using the 2.6-m Shain telescope (wide $R-0.5-$ 0.75 micron band with 4 -s exposures, hereafter wR) and the 1.25-m AZT-11 telescope (UBVRI photometric bands with 16-s time resolution) of the Crimean Astrophysical Observatory during 1990-1999 (see Table 1). The current instrumental UBVRI bands slightly differ from the standard Johnson system and are the same as that used by Piirola et al. (1994). These observations cover a wide energy range and allow us to investigate different sources and mechanisms of variability caused by accretion in magnetic cataclysmic variables. The total number of data points is 19025 , which were obtained during 22 nights of observations. The data have been obtained as described by Piirola et al. (1994). The timings of observations were reduced

Table 1. Log of photopolarimetric observations of QQ Vul.

\begin{tabular}{|c|c|c|c|c|c|}
\hline $\begin{array}{l}\text { Data } \\
{[\mathrm{Y} / \mathrm{M} / \mathrm{D}]}\end{array}$ & $\begin{array}{l}\text { Julian } \\
\text { data } \\
\text { JD } 24 \ldots\end{array}$ & $\Delta T, d$ & $\begin{array}{c}\Delta T \\
\text { phase } \\
\text { coverage }\end{array}$ & $\bar{N}$ & Tel. \\
\hline $90 / 08 / 17$ & 48121.29 & 0.18 & 1.16 & 416 & $1.25-\mathrm{m}$ \\
\hline $90 / 08 / 20$ & 48124.30 & 0.11 & 0.73 & 247 & $1.25-\mathrm{m}$ \\
\hline $92 / 07 / 29$ & 48833.49 & 0.04 & 0.24 & 95 & $1.25-\mathrm{m}$ \\
\hline $92 / 07 / 30$ & 48834.30 & 0.10 & 0.67 & 251 & $1.25-\mathrm{m}$ \\
\hline $92 / 07 / 31$ & 48835.33 & 0.09 & 0.60 & 287 & $1.25-\mathrm{m}$ \\
\hline $92 / 08 / 28$ & 48863.28 & 0.17 & 1.12 & 470 & $1.25-\mathrm{m}$ \\
\hline $92 / 08 / 30$ & 48865.25 & 0.20 & 1.30 & 633 & $1.25-\mathrm{m}$ \\
\hline $94 / 08 / 10$ & 49575.29 & 0.11 & 0.74 & 2048 & 2.6-m \\
\hline $94 / 08 / 10$ & 49575.35 & 0.07 & 0.43 & 364 & $1.25-\mathrm{m}$ \\
\hline $94 / 08 / 11$ & 49576.28 & 0.06 & 0.40 & 127 & $1.25-\mathrm{m}$ \\
\hline $94 / 09 / 05$ & 49601.26 & 0.16 & 1.01 & 400 & $1.25-\mathrm{m}$ \\
\hline $94 / 10 / 04$ & 49630.22 & 0.15 & 0.97 & 342 & $1.25-\mathrm{m}$ \\
\hline $95 / 09 / 25$ & 49986.28 & 0.14 & 0.90 & 294 & $1.25-\mathrm{m}$ \\
\hline $95 / 09 / 26$ & 49987.40 & 0.02 & 0.12 & 31 & $1.25-\mathrm{m}$ \\
\hline $95 / 09 / 27$ & 49988.26 & 0.03 & 0.22 & 59 & $1.25-\mathrm{m}$ \\
\hline $97 / 10 / 04$ & 50726.35 & 0.05 & 0.33 & 960 & 2.6-m \\
\hline $97 / 10 / 05$ & 50727.20 & 0.13 & 0.85 & 1152 & 2.6-m \\
\hline $98 / 05 / 24$ & 50958.46 & 0.07 & 0.47 & 1280 & 2.6-m \\
\hline $98 / 05 / 25$ & 50959.39 & 0.14 & 0.92 & 2384 & 2.6-m \\
\hline $98 / 08 / 17$ & 51043.28 & 0.18 & 1.14 & 1021 & $1.25-\mathrm{m}$ \\
\hline $98 / 08 / 17$ & 51043.32 & 0.21 & 1.34 & 3584 & 2.6-m \\
\hline $99 / 08 / 11$ & 51402.40 & 0.15 & 0.99 & 2592 & 2.6-m \\
\hline
\end{tabular}

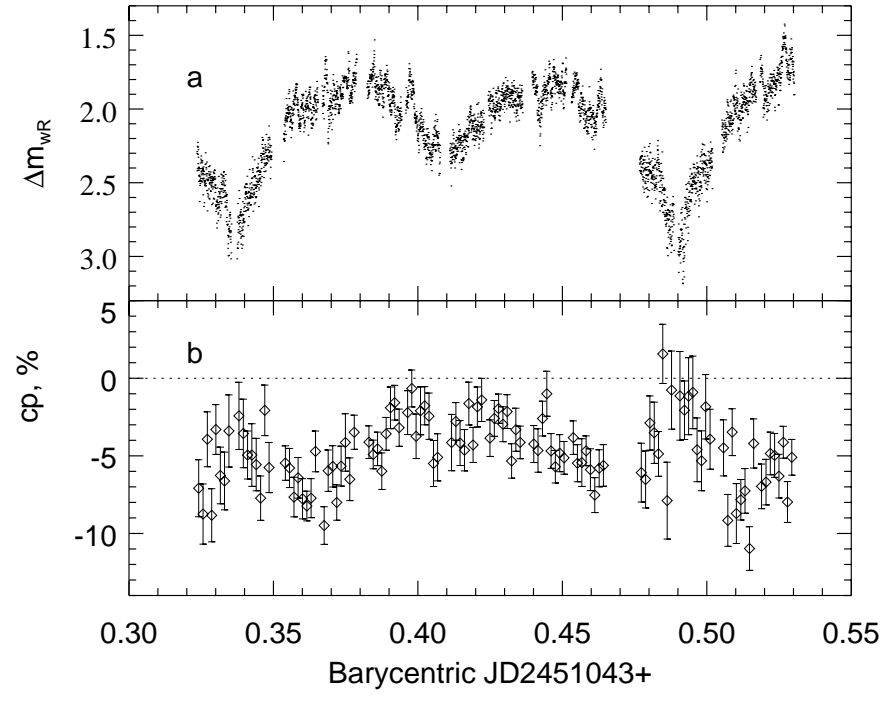

Fig. 1. Example of photometric a) and circular polarimetric b) curves of QQ Vul obtained using 2.6-m Shain telescope.

to the solar system's barycenter using the formulae by Soma et al. (1988).

Despite the instability of individual light curves, typical patterns were observed during each nights. For each orbital period, the primary minimum was always observed, which has amplitude of up to $1^{\mathrm{m}}$. The secondary minimum was observed at the phase 0.5 (if we take the primary minimum as the zero phase) and its depth reaches 0.5 (see Fig. 1a). The circular polarization curve was binned into $64 \mathrm{~s}$ intervals (see Fig. 1b). The circular polarization accuracy estimates obtained from quantum statistics are smaller than the real deviations (Shakhovskoy et al. 1998). This implies the presence of polarization variations with a characteristic time being smaller than the $64 \mathrm{~s}$ integration time bins. During the primary minimum, the circular polarization is close to $0 \%$. However, during the secondary minimum, quite the contrary, it sharply increases to about $-4 \%$. One maximum of circular polarization is observed 0.025 day $=0$ p .16 after the primary minimum and another one occurs about $0^{\mathrm{d}} .015$ day $=0^{\mathrm{p}} .096$ before it.

In Fig. 2, we present profiles of the main minimum of QQ Vul for different epochs. The top two plots were obtained during subsequent nights. During this time, the accretion geometry had already changed. In Table 2 , the timings of primary minima from our observations of QQ Vul are listed. There is a significant decrease in the amplitude of the primary minima going from $U$ to $I$ data. The primary minimum in $I$ became asymmetric during the last night (JD 2451043 ) of the $1.25-\mathrm{m}$ telescope observations (Fig. 3). The system becomes redder at the brightness minima.

\section{Analysis of observations}

\subsection{Photometric period}

Some quasi-periodic period changes in magnetic cataclysmic variables can be described by asynchronous or quasisynchronous rotation of the white dwarf. These processes have a time-scale of years (Andronov 1987). However, the mass and 


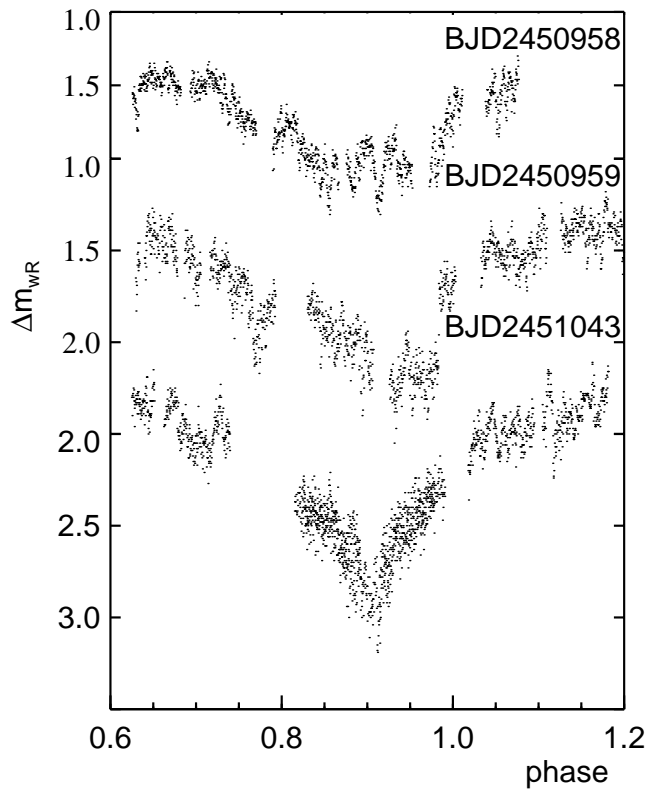

Fig. 2. Primary minima profiles for different observation nights of QQ Vul obtained with the 2.6-m telescope. Phases are calculated using ephemeris from Eq. (1).

Table 2. Timings of primary minima of QQ Vul.

\begin{tabular}{ccr}
\hline \hline Min JD 24... & $\sigma_{\min }$ & Telescope \\
\hline 48121.3632 & 0.0021 & $1.25-\mathrm{m}$ \\
48835.3872 & 0.0012 & $1.25-\mathrm{m}$ \\
48863.3558 & 0.0013 & $1.25-\mathrm{m}$ \\
48865.3659 & 0.0020 & $1.25-\mathrm{m}$ \\
49601.3464 & 0.0020 & $1.25-\mathrm{m}$ \\
50958.5023 & 0.0018 & $2.6-\mathrm{m}$ \\
50959.4342 & 0.0012 & $2.6-\mathrm{m}$ \\
51043.3356 & 0.0009 & $2.6-\mathrm{m}$ \\
51043.3367 & 0.0012 & $1.25-\mathrm{m}$ \\
51043.4893 & 0.0012 & $2.6-\mathrm{m}$ \\
51402.4438 & 0.0013 & $2.6-\mathrm{m}$ \\
\hline
\end{tabular}

momentum transfer can also be observable as secular period changes.

The photometric period of the QQ Vul is determined by the timings of the primary minima. Because the primary minima are explained as a combination of a self-eclipse of the accretion column and the cyclotron beaming effect, the timings allow to determine a spin period of the white dwarf (assuming the column is not migrating on the star) rather than the orbital period. If the phase of the primary minima may have been displaced due some processes in the system, the character of these shifts may allow to study such processes.

For the investigation of such possible photometric period changes, we have used 87 timings of minima, collected by Andronov \& Fuhrmann (where are 2 determined by Nousek et al. 1984 and 1 obtained by Mukai et al. 1986). Also we have used 20 timings, obtained by Halevin et al. (1997) from photographic observations. From the observations obtained at the AZT-11, we have determined 6 minima in $B$ band using the parabolic fits for the data near minima. Despite the fact that the observations from the Shain telescope were obtained

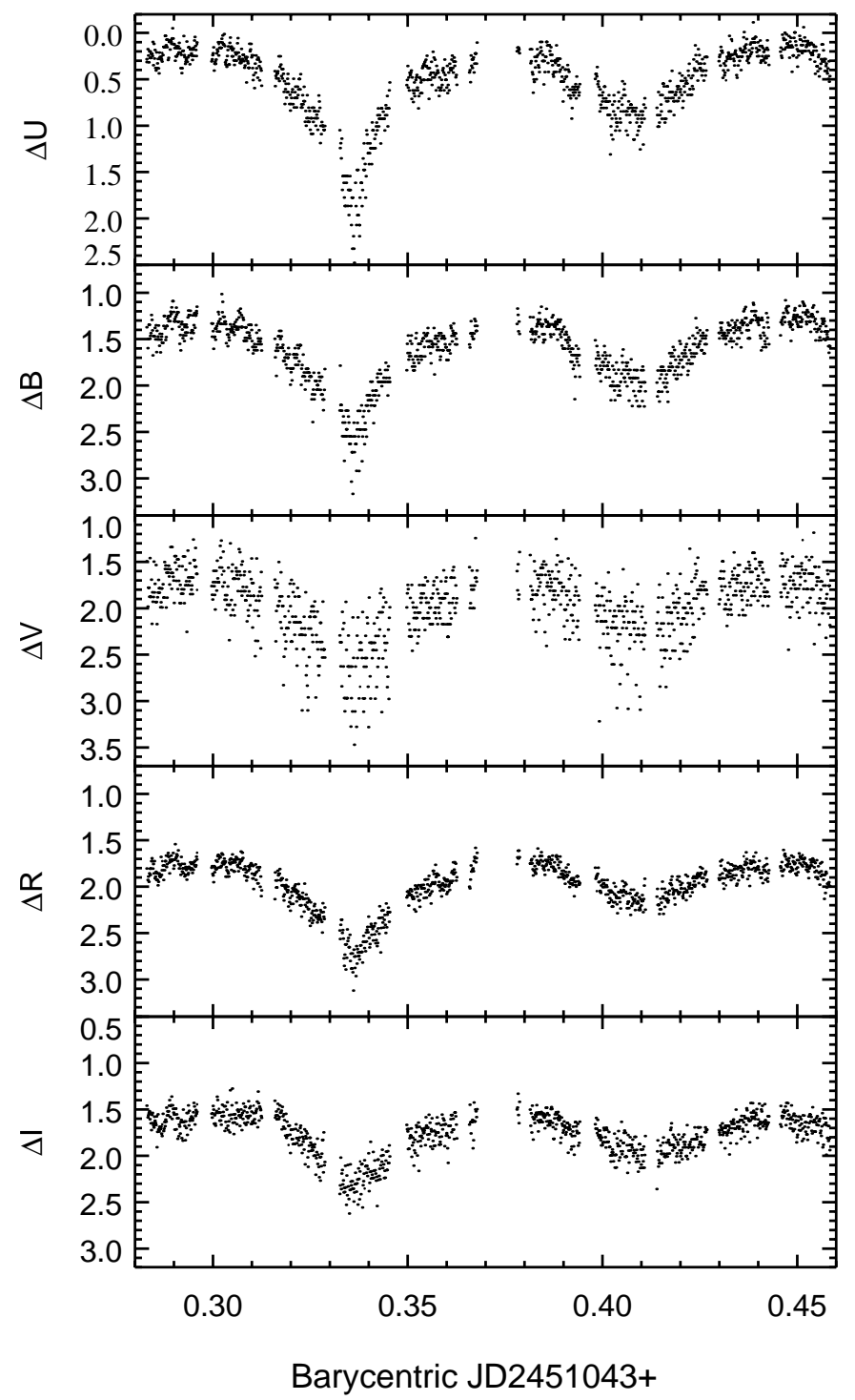

Fig. 3. Photometric UBVRI curves of QQ Vul obtained using $1.25-\mathrm{m}$ telescope on JD 2451043.

in the wR filter, we have used those 5 minima timings together with those obtained at other telescopes in the $B$ and pg systems. We have found no statistically significant phase shift from the simultaneous observations obtained at the AZT-11 and Shain telescopes. Altogether, these data cover more than 60 years (from 1934). The resulting phase shift curve of primary minima is presented in Fig. 4.

The phase-shift diagram was computed using the ephemeris found by Schwope et al. (2000):

HJDSp $=2448446.47105(48)+0.15452011(11) \cdot E$.

Here the numbers in brackets indicate statistical error estimates in units of the last decimal digit. The "zero" phase corresponds to an inferior conjunction of the secondary star, i.e. to the orbital motion.

The dependence of the phase shift on time is well represented by a linear function, so we propose a combined updated ephemeris

$\mathrm{HJD}=2448446.47105(48)+0.15452040(2) \cdot E$ 


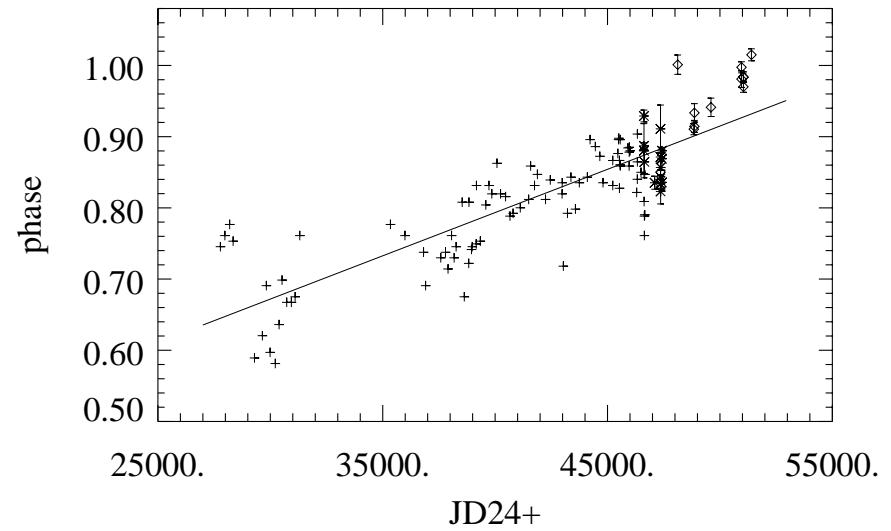

Fig. 4. Phases of timings of the primary minimum of QQ Vul, calculated using the ephemeris presented by Schwope et al. (2000). The "zero" phase corresponds to an inferior conjunction of the secondary star. Here the crosses are the data collected by Andronov \& Fuhrmann, the asterisks are the timings of Halevin et al. (1997) and the rhombuses correspond to the Crimean data, which are listed in Table 2. The fit corresponds to the our photometric ephemeris (Eq. (2)). All points, excluding the 2.6-m telescope observations ( $\mathrm{w} R$ band), have been computed using observations in B or pg photometric systems.

which keeps the phase zero defined by the orbital motion, and uses value of the photometric period. The initial epoch for the photometric minima is HJD $2440000.06166 \pm 0.00074$, so the spectroscopic conjunction occurs $0.0989 \pm 0.0048$ after the photometric minima.

The systematic phase shift of the primary minimum, according to the ephemeris (1), reaches $\sim 0.4$ of the period. The physical interpretation is improbable without the presence of dramatic changes of the accretion geometry, and, as a result, of the shape of the optical light curve. This apparent trend vanishes, if using the ephemeris (2). As the orbital period coincides with the spin period of the white dwarf, what is characteristic for polars, we suggest the period value in Eq. (2) corresponds both to the orbital motion and the spin of the white dwarf.

\subsection{Search for possible quasi-periodic oscillations using the wavelet analysis}

Many papers are devoted to an application of the wavelet method to periodic or multiperiodic processes (Goupil et al. 1991; Szatmary et al. 1994; Fritz \& Bruch 1998). In this section we show how the wavelet maps of the irregular variability may be interpreted.

Variations at multiple time-scales, which are usually detected in AM Her type stars, are most likely explained by separate mechanisms. Short variations with a time-scale of about 2-5 min can be explained by a sequence of different small flares. The quasi-periodic oscillations (QPOs) with time-scales of about 5-10 min have similar frequencies as the oscillations of the ionization front near the $L_{1}$ point (King 1989). Also some light curve variations with time-scales of several minutes can be interpreted as a possible result of the magneto-acoustic oscillations of the white dwarf's surface (Lou 1995).

To search for the possible QPOs at time-scales of about 5-20 min, we have used the observations obtained at the 2.6-m

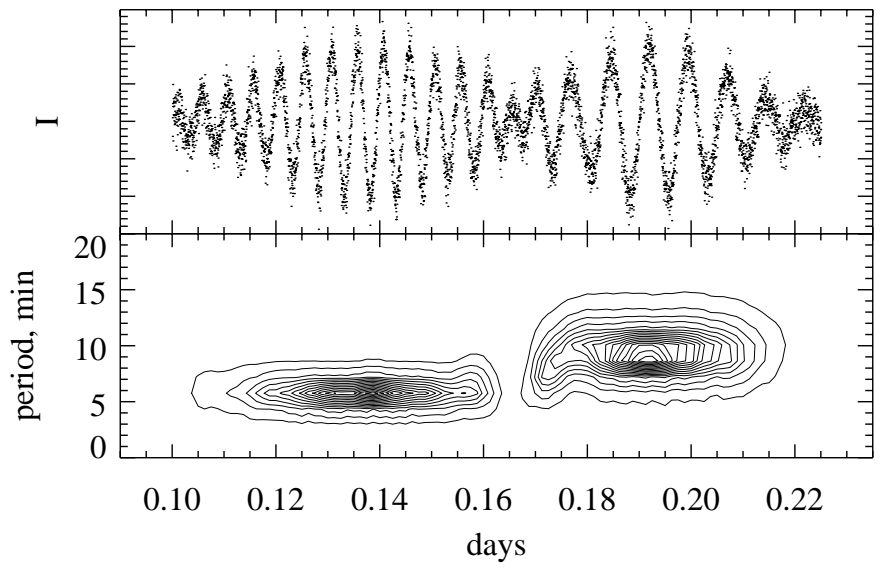

Fig. 5. Model of the quasi periodical variability for sequence of two events with periods of 7.2 and $10.8 \mathrm{~min}$ and a corresponding wavelet map. Note, that the test-function WWZ (Weighted Wavelet Ztransform, Foster 1996) has maximum at the period slightly exceeding that of the signal.

Shain telescope, because its 4-s time resolution is better than that of the $1.25-\mathrm{m}$ telescope. The orbital photometrical variations have been smoothed by using the method of running parabola (Andronov 1997). The optimal value of the filter halfwidth $\Delta t=0.055$ has been determined from maximization of the "signal-to-noise" ratio.

To avoid apparent effects of low-frequency trends on the test-functions at high frequencies, the original data have been detrended, i.e. the running parabola fit was subtracted from the observations. For these detrended time series, the test functions have been computed using the code described by Andronov (1994a).

For visualization, we have used the Weighted Wavelet Z-transform (WWZ) test-function (Foster 1996), which is characterized as having the best contrast among other test functions. However, the values of time-scales have been calculated using the $S$ test-function introduced by Andronov (1998). Contrary to the test-function WWZ, the maximum of the test-function $S$ occurs at an unbiased frequency value.

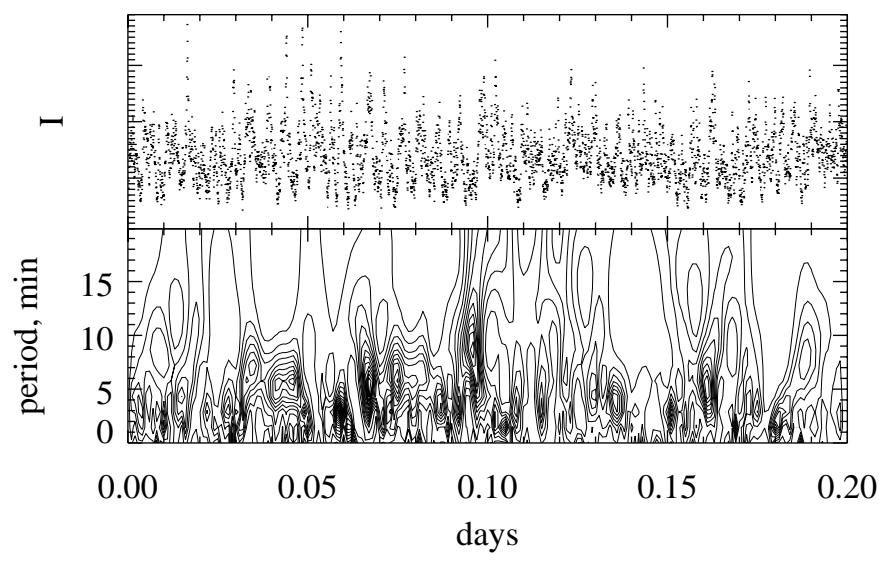

Fig. 6. Model of the purely flare variability with duration of single flare of about $25 \mathrm{~s}$ (up) and a corresponding wavelet map (bottom). 


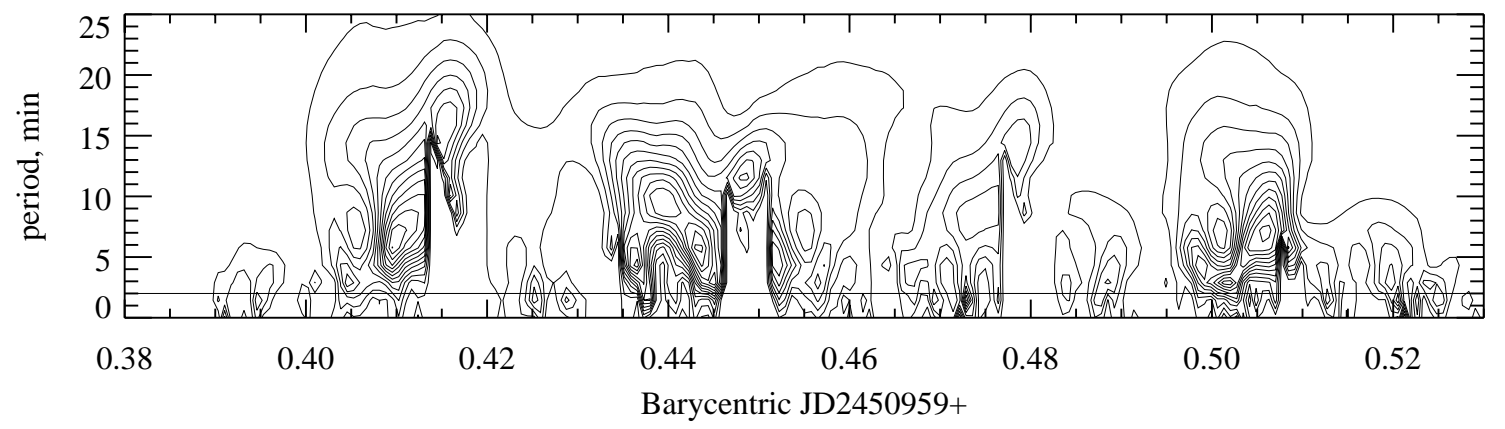

Fig. 7. Example of the wavelet map for observations of QQ Vul obtained using the 2.6-m telescope. The horizontal line corresponds to a 2-min peak in the distribution of QPOs (Fig. 8) and the power spectrum (Fig. 9).

To illustrate the capability of the wavelet analysis to determine character of the variability, we have generated signals using two models for the cases of the presence: a) QPOs on light curves and b) purely flare variability.

In Fig. 5 one can see a model for two quasi-periodical events with waves with time-scales (periods) of 7.2 and $10.8 \mathrm{~min}$, one of which has replaced another. The corresponding dependence of the test-function WWZ, on time and trial period (often called "the wavelet map") is shown at the bottom of Fig. 5. For such quasi-periodical events, using the wavelet map, it is easy to determine their duration (coherence time). For our sample, they are equal to about 0.06 and 0.05 , i.e. about 12 and 7 oscillation periods, respectively (see Otazu et al. 2002 for more detailed discussion).

Figure 6 shows the wavelet map (bottom) for purely flare model curve (top). The flares have an exponential profile with a time-scale about $25 \mathrm{~s}$. In total, 5000 such overlapping events are present at the model curve. As is expected, there are no detected events with a coherence time-scale larger than their period of variations.

So one can see that for totally non-coherent data, the peaks at the wavelet map are characterized by the apparent duration of the characteristic time-scale of variability.

An example of a wavelet map for observations of QQ Vul is shown in Fig. 7.

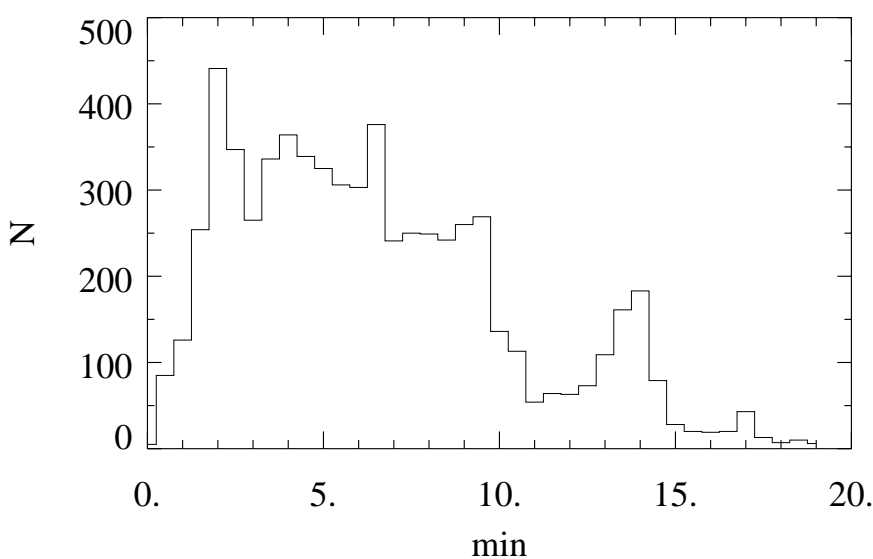

Fig. 8. The distribution of the time-scales (or the periods, if assuming the QPOs) of photometrical variations, determined using wavelet analysis.

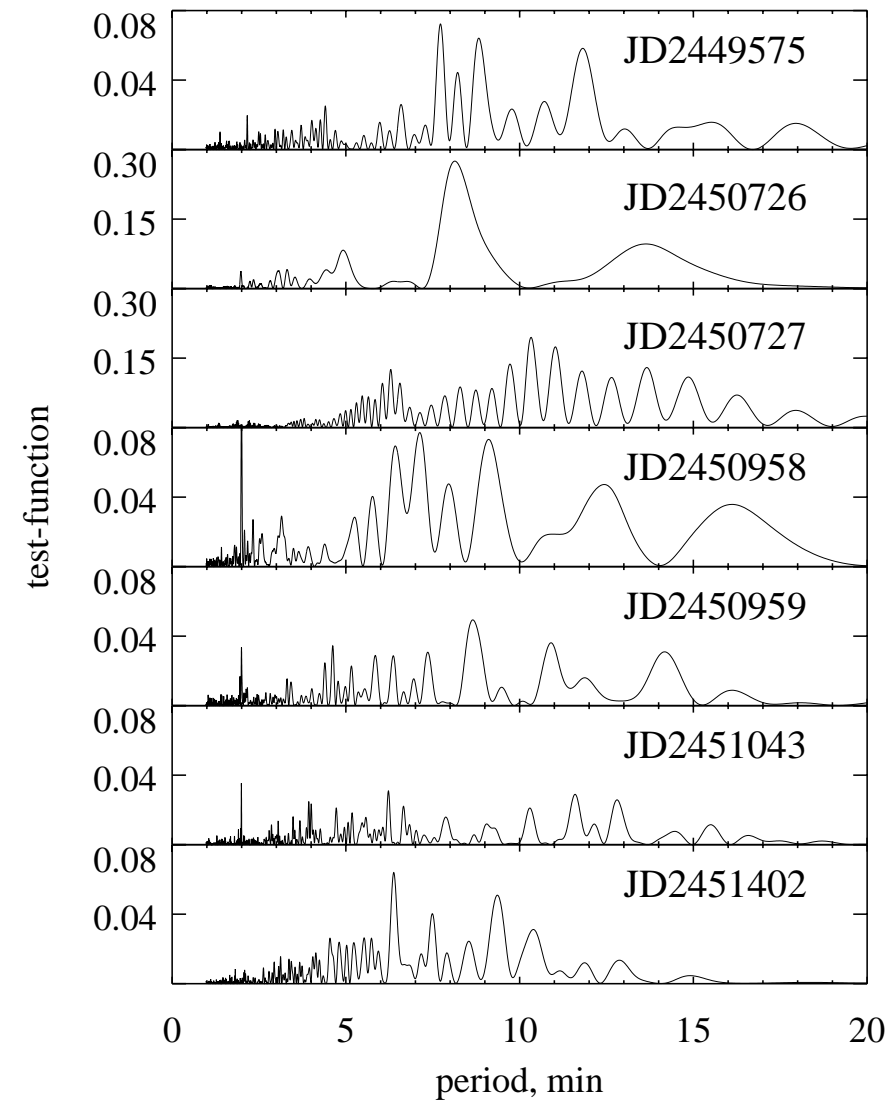

Fig. 9. Power spectrum for individual nights of 2.6-m telescope observations. The test function is the ratio "signal-to-(signal+noise)" (Andronov 1994b).

Using the local parabolic approximations of the wavelet test-functions, we have calculated the time-scales of light curve events. The distribution of their values is presented in Fig. 8. One can see that the maximum of the distribution occurs at about 2 min.

Because the trend has been removed from the data, all variations longer than 20 min have been discarded and cannot be studied.

For comparison, we have computed the power spectrum (using the method of Fourier analysis, which was described by Deeming 1975) for the observations obtained at the 2.6-m telescope (Fig. 9). Contrary to the wavelet analysis, 


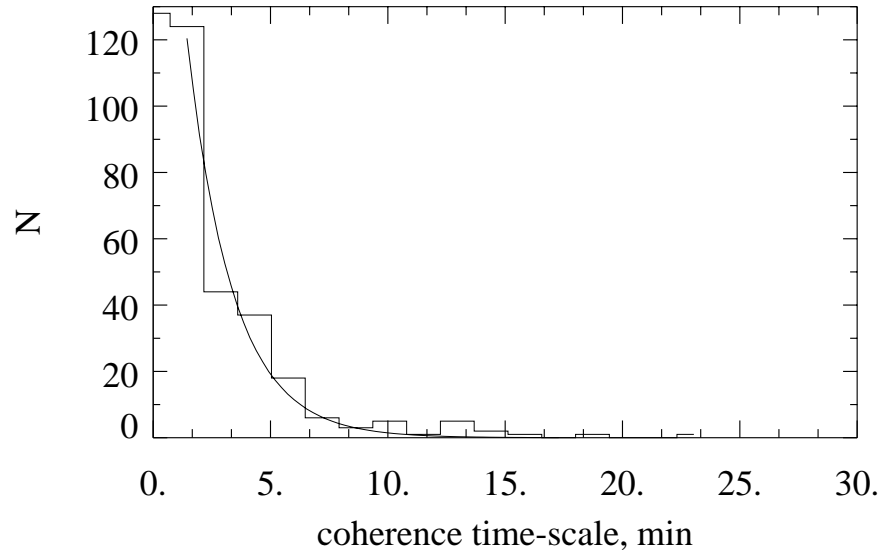

Fig. 10. The distribution of the coherence time-scales for photometrical events (for QPOs it is a time interval, during which they are observed). The solid line shows an exponential decay with a best fit value $\tau=1.79 \mathrm{~min}$.

which is sensitive both to coherent and non-coherent oscillations, the power spectrum indicates either the presence of oscillations with large coherence times, or the apparently periodic, randomly repeating pulses. Therefore, the detected time-scales obtained using these different methods do not generally coincide. As one may conclude from the night-to-night shifts of the peaks, there are no coherent oscillations in the variations of QQ Vul.

We note the detection of the peaks corresponding to periods of about $2 \mathrm{~min}$ for the observations on JD 2450958 , JD 2450959 and JD 2451 043. Such a time-scale corresponds to the characteristic time between well resolved single flares. But this process is not coherent and cannot be resolved on a simple power spectrum.

The calculations of the coherence time for the light curve events have allowed us to conclude that in most cases there are no events with a duration significantly longer than the timescale of the variations (see Figs. 10 and 11). Hence, we can say that in this case a simple power spectrum analysis is not effective.

Taking into account the non-coherent character of detected events, we can interpret the estimated time-scales as a typical time interval between subsequent prominent non-overlapping flares (or any another instability manifestations). Using the exponential approximation $f=A \cdot \mathrm{e}^{-x / \tau}$ for the distribution function of the time-scales (solid line in Fig. 10), we have determined the value of $\tau=1.79 \pm 0.20 \mathrm{~min}$, which characterizes a mean time between consecutive flares. Such an exponential distribution is expected, if the flares are randomly distributed in time.

A search of the dependencies between the time-scales of the events at the light curve and the brightness and circular polarization of the system does not show any statistically significant results.

\subsection{Shot noise}

Fast variability at a time-scale of dozens of seconds is well described by a shot noise model with an exponential decay of its

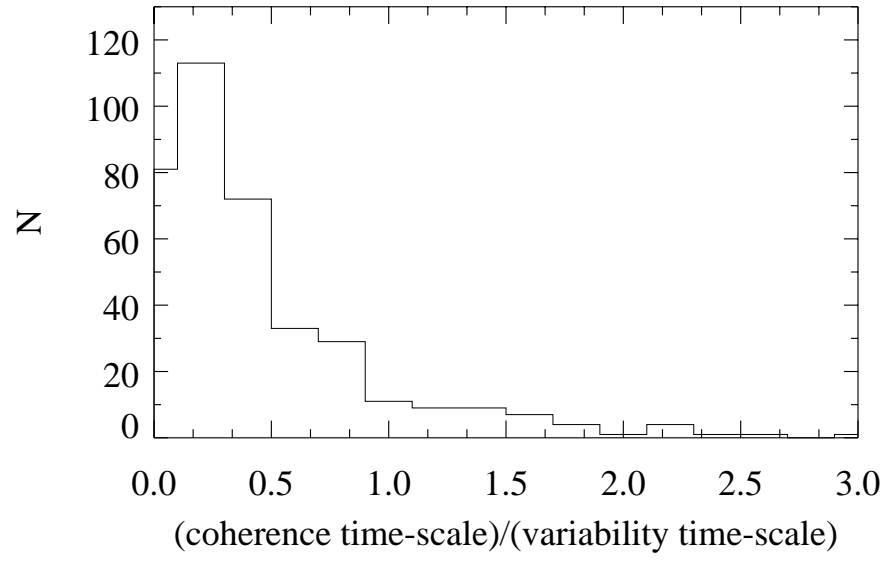

Fig. 11. The distribution of the ratio between the duration of the event (the coherence time-scale) and the variability time-scale (period in the case of QPOs) for photometrical events.

auto-correlation function (ACF, Andronov 1994a). The shot noise is interpreted as being the result of accretion of large diamagnetic blobs (Beardmore \& Osborne 1997; Kuijpers \& Pringle 1982; Panek 1980). Possible differences between the location of the coupling points for blobs with different sizes (Wynn \& King 1995) cause the variability of the shot noise decay time.

To search for this effect, we have removed the long-term orbital variability from the light curves of QQ Vul. The third order polynomial approximation for each uniform section of the run was used. In Fig. 12, the light curve is shown, obtained using the 2.6-m Shain telescope with a time resolution of $4 \mathrm{~s}$. The solid lines are the 3rd order approximations for a continuous part of the run. Below the corresponding detrended curve is placed (i.e. the residuals from the fit). We have subdivided such curves into uniform runs, and for each one, we have calculated the ACF.

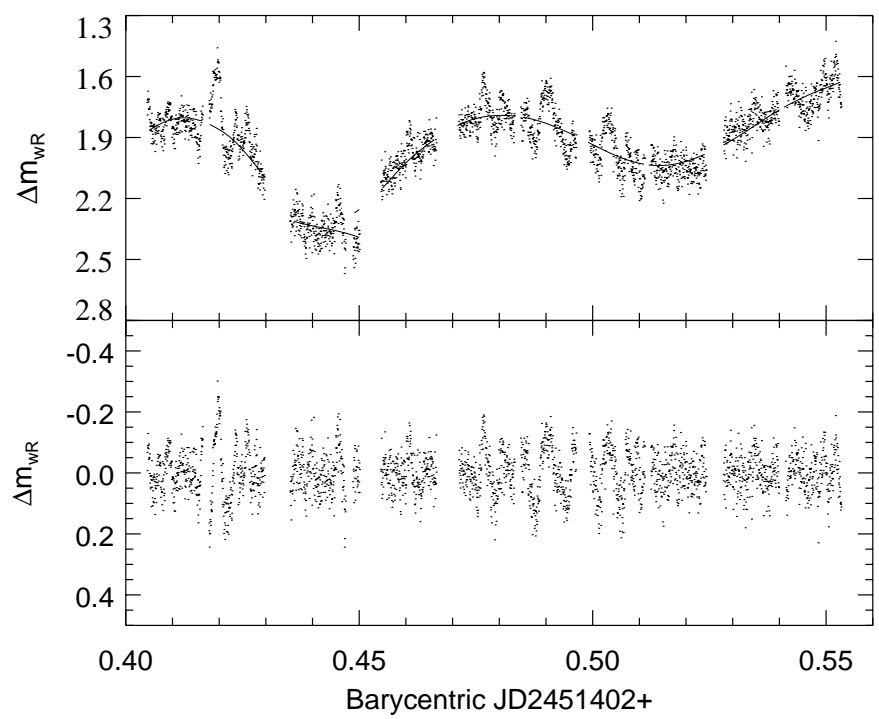

Fig. 12. An example of the original (top) and locally detrended (bottom) photometric data for QQ Vul, obtained using 2.6-m Shain telescope on JD 2451402. 


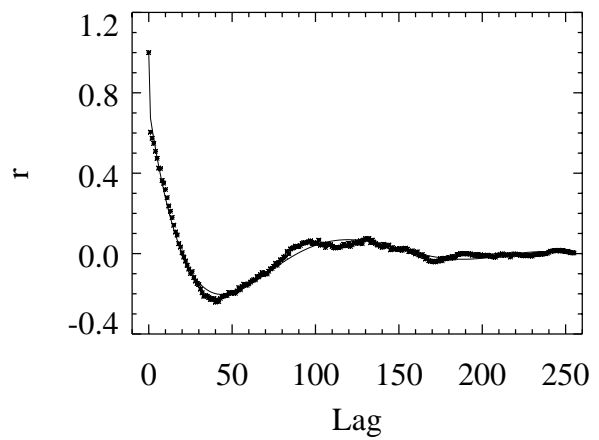

Fig. 13. An example of an auto-correlation function (ACF). The thick solid line corresponds to the ACF for an auto-regression first-order model with best fits values of the parameters. The lag is expressed in units of time resolution (4s).

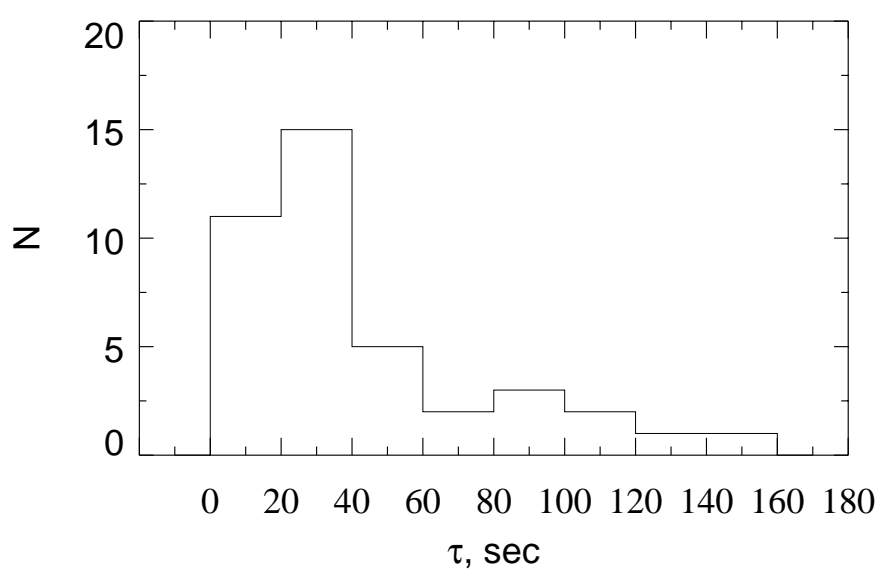

Fig. 14. The distribution of the shot noise exponential decay time for the 2.6-m telescope' observations of QQ Vul.

The shot noise decay time is determined from the autocorrelation function using the method by Andronov (1994a) taking into account the bias due to the finite length of the run and the trend removal.

In total, we have determined 40 shot noise decay time values from the 2.6-m telescope observations, and 125 ones (taking into account five colors) by using the AZT-11 observations. In Fig. 13, an example of the mean ACF is shown, which has been computed using the run of 256 points. The thick solid line corresponds to the first-order auto-regressive model which takes into account the finite length of the run and the fact, that the polynomial trend was removed.

In Fig. 14, the histogram of the values of the decay timescale, $\tau$, for the 2.6-m telescope observations is shown. The maximum of the distribution lies at about $25 \mathrm{~s}$, and the mean value is $26.8 \mathrm{~s}$.

In Fig. 15 we present curves showing the exponential decay time of the shot noise. Plots $a$ and $b$ are typical orbital curves of brightness and circular polarization (for JD 2451043 ). Plot c gives the exponential decay time. Despite a large scatter of the points, there are several regularities in these curves. The maximal values of $\tau$ correspond to phase zero, when the linear polarization is at its maximum and the accretion region is located near the limb of the white dwarf.

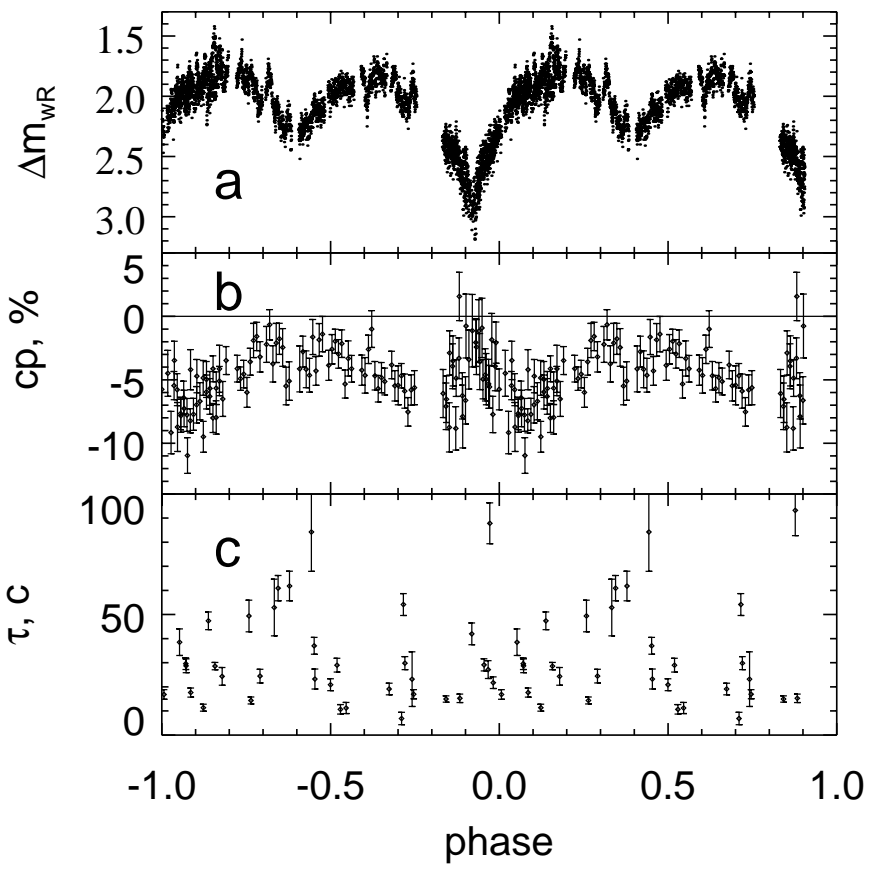

Fig. 15. Phase variations of the shot noise exponential decay time c). Phases are calculated using the ephemeris from Eq. (1). For comparison, they are shown together with the photometrical a) and polarimetrical b) curves. The data is shown twice for clarity.

Thus, in this section we have found that the fast variability of QQ Vul is well described by the shot noise model, i.e. a result of superposition of flares with a time-scale of about $25 \mathrm{~s}$. If we accept an interpretation of the shot noise variability as a result of the blobby accretion on to the white dwarf's atmosphere, then the time-scale of $25 \mathrm{~s}$ gives us the mean falling time of the blobs. In the Discussion section, we introduce a technique for determination of the linear sizes of the blobs using their falling time.

\section{Discussion}

Some parameters of the accretion flow blobs can be estimated from investigation of the shot noise or resolved single flares. If $\tau$ is the effective emitting time for a falling blob with a length $l$ (Beardmore \& Osborne 1997), then $l=v_{\mathrm{ff}} \tau / 4$, where $v_{\mathrm{ff}}$ is the free-fall velocity above the shock. Using some assumptions about the structure of the magnetic field, one can determine some parameters of the blobs.

Such blobs originate due to the Rayleigh-Taylor instabilities, when a heavy fluid (matter) is opposed in the gravitation field to a light fluid (magnetic field, Lang 1974). According to Hameury et al. (1986), the minimal scale of instabilities is

$\lambda=\frac{B}{2 g \rho_{\mathrm{b}}}=\frac{B^{2} r_{\mathrm{b}}^{2}}{2 G M_{\mathrm{wd}} \rho_{\mathrm{b}}}$,

where $G$ is the gravitational constant, $B$ is a magnetic field intensity, $g$ is the acceleration due to gravity at the distance $r_{\mathrm{b}}$ from the white dwarf, $M_{\mathrm{wd}}$ is a mass of the white dwarf and $\rho_{\mathrm{b}}$ is the blob density. But this mechanism takes place only when 


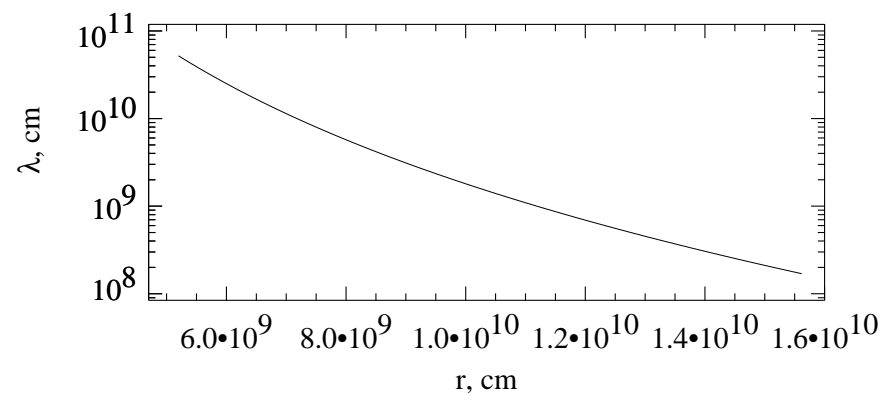

Fig. 16. The minimal Rayleigh-Taylor unstable scale (Eq. (3)) for an accretion flow in QQ Vul for an accretion rate $10^{17} \mathrm{~g} / \mathrm{s}$ as a function of distance from the white dwarf. We take the strength of the magnetic field at the poles to be $30 \mathrm{MG}$ (here we assumed the same value as Cropper 1998).

the magnetic pressure $p_{B}$ becomes larger than the gas thermal pressure $p_{\mathrm{T}}$.

Using the algorithm described by Heerlein et al. (1999), we have determined the pressure in the flow, the mean coupling radius and have calculated the smallest unstable length as a function of the distance (Fig. 16).

During the fall onto the white dwarf's (WD below) surface, the blobs are deformed owing to tidal forces and the magnetic field. The length of a deformed blob near the shock above the WD's surface is determined as (here we used statement $l \sim$ $\left(r / R_{\mathrm{wd}}\right)^{1 / 2}$ from Beardmore \& Osborne 1997)

$l=\lambda\left(\frac{r_{\text {coup }}}{R_{\mathrm{wd}}+h_{\mathrm{col}}}\right)^{1 / 2}$,

where $r_{\text {coup }}$ is the coupling distance, $R_{\mathrm{wd}}$ is the radius of the white dwarf and $h_{\text {col }}$ is the height of the accretion column above the WD's surface.

But the blob length $l$ also is evaluated as

$l=\tau v_{\mathrm{ff}} / 4=\frac{\tau}{4}\left(\frac{2 G M_{\mathrm{wd}}}{R_{\mathrm{wd}}+h_{\mathrm{col}}}\right)^{1 / 2}$,

where the factor of $1 / 4$ arises from the strong shock conditions. Thus

$\lambda=\frac{\tau}{4}\left(\frac{2 G M_{\mathrm{wd}}}{r_{\text {coup }}}\right)^{1 / 2}$.

A very interesting result is that the falling time of the blob does not depend on the stopping height. So the emissivity time for single blobs is determined only by the initial size and by the coupling distance from the center of the white dwarf.

Using the formula for the coupling distance (Ferrario et al. 1999) we have found that approximately $r_{\text {coup }} \sim M_{\mathrm{wd}}^{-8 / 21}$, and, hence $\lambda \sim M_{\mathrm{wd}}^{37 / 42}$. So, the calculated values of the initial sizes of the blobs are sensitive to uncertainties in the determination of the mass of the white dwarf.

More precise calculations of the effects which have an influence on the formation of the flow instabilities are necessary using the MHD radiative modelling of the accretion flow and its Rayleigh-Taylor instabilities, taking into account either the dipole or quadrupole structure of white dwarf magnetic field.

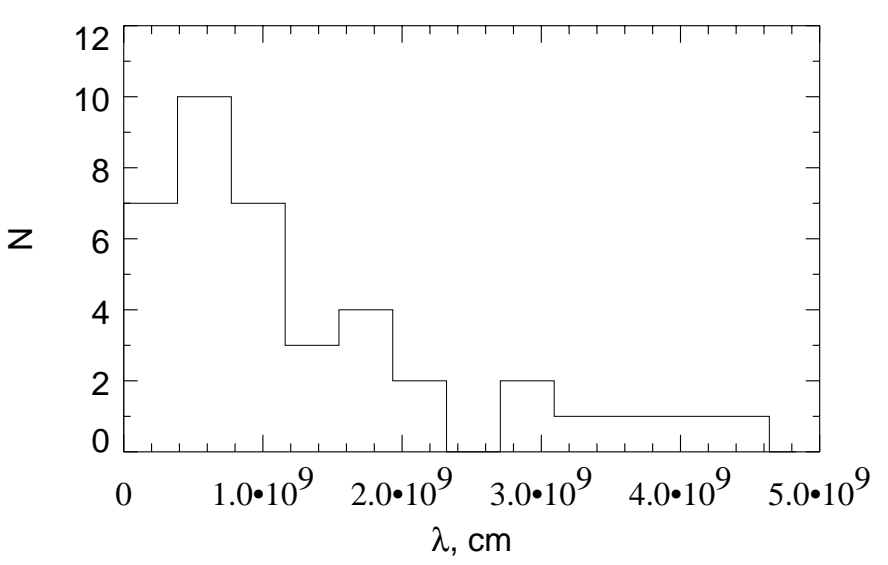

Fig. 17. The distribution of the linear sizes of non-deformed blobs in an accretion flow of QQ Vul for an accretion rate $10^{17} \mathrm{~g} / \mathrm{s}$ and a coupling distance $5.1 \times 10^{9} \mathrm{~cm}$.

For preliminary calculations, we have used Eq. (6) described above. For example, if we fix the value of the coupling point distance, then from the observed distribution of $\tau$ we can calculate linear blob sizes before their deformation by the magnetic field. Such an assumption is not so strong because the coupling process acts only along a short part of the flow trajectory.

Thus, for the mass flow rate of $10^{17} \mathrm{~g} / \mathrm{s}$ and the WD's mass value of $0.54 M_{\odot}$ (Catalan et al. 1999), the coupling distance is about $5.1 \times 10^{9} \mathrm{~cm}$. Using the mass-radius relation for the white dwarfs in a form published by Andronov \& Yavorskij (1990) (see also Nauenberg 1972), the radius, which corresponds to the mass of $0.54 M_{\odot}$, is equal to $R_{\mathrm{wd}}=7.42 \times 10^{8} \mathrm{~cm}$. So the coupling occurs at $\sim 7 R_{\mathrm{wd}}$. The corresponding distribution of the initial sizes of the blobs for QQ Vul is shown in Fig. 17. The maximum of the distribution occurs near $6 \times 10^{8} \mathrm{~cm}$, which corresponds to formation of the blobs at a distance of $1.2 \times$ $10^{10} \mathrm{~cm}$ from the center of the white dwarf (Fig. 16).

\section{Conclusions}

As a result of our investigations, we have determined the sizes of blobs in the accretion flow $\left(\sim 6 \times 10^{8} \mathrm{~cm} \simeq 0.8 R_{\mathrm{wd}}\right)$.

Having a negative result in detecting QPOs, and having good fits using the auto-correlation analysis, we report: no detectable QPOs, but definite "shot noise".

By interpreting the shot time-scale events (detected using the wavelet analysis) as a sequence of single flares, we have determined that the effective time delay between such neighboring most prominent events is $\sim 1.8 \mathrm{~min}$. Another result is the more precise determination of the photometric period of this system.

Acknowledgements. We thank the anonymous referee for a critical reading of this paper and many useful comments. We also thank Axel Schwope and Paul A. Mason for interesting discussions and for comments on the manuscript. 


\section{References}

Andronov, I. L. 1987, Astrophys. Space Sci., 131, 557

Andronov, I. L. 1994a, Astron. Nachr., 315, 353

Andronov, I. L. 1994b, Odessa Astron. Publ., 9, 49

(http://oap09.pochtamt.ru)

Andronov, I. L. 1997, A\&AS, 125, 207

Andronov, I. L. 1998, Kinemat. Phys. Celest. Bodies, 14, 490

Andronov, I. L., \& Fuhrmann, B. 1987, IBVS, 2976, 1

Andronov, I. L., \& Yavorskij, Yu. B. 1983, Soviet Astron. Lett., 9, 291

Andronov, I. L., \& Yavorskij, Yu. B. 1990, Contrib. Astron. Obs. Skalnate Pleso, 20, 155

Beardmore, A. P., \& Osborne, J. P. 1997, MNRAS, 290, 145

Beardmore, A. P., Ramsay, G., Osborne, J. P., et al. 1995, MNRAS, 273, 742

Catalan, M. S., Schwope, A. D., \& Smith, R. C. 1999, MNRAS, 310, 123

Cropper, M. 1998, MNRAS, 295, 353

Deeming, T. J. 1975, Ap\&SS, 36, 137

Ferrario, L., \& Wehrse, R. 1999, MNRAS, 310, 189

Foster, G. 1996, AJ, 112, 1709

Fritz, T., \& Bruch, A. 1998, A\&A, 332, 586

Goupil, M. J., Auvergne, M., \& Baglin, A. 1991, A\&A, 250, 89

Halevin, A. V., Andronov, I. L., Kolesnikov, S. V., \& Kimeridze, G. N. 1997, Odessa Astron. Publ., 10, 25

(http://oap10.pochtamt.ru)

Hameury, J. M., King, A. R., \& Lasota, J.-P. 1986, MNRAS, 218, 695

Heerlein, P., Horne, K., \& Schwope, A. D. 1999, MNRAS, 304, 145

King, A. R. 1989, MNRAS, 241, 365
Kuijpers, J., \& Pringle, J. E. 1982, A\&A, 114, L4

Lang, K. R. 1974, Astrophysical formulae (Springer-Verlag)

Lou, Y. Q. 1995, MNRAS, 275, L11

McCarthy, P., \& Bowyer, S. 1986, ApJ, 311, 873

Meggitt, S. M. A., \& Wickramasinghe, D. T. 1984, MNRAS, 207, 1

Mukai, K. 1988, MNRAS, 232, 175

Mukai, K., Bonnet-Bidaud, J.-M., Charles, P. A., et al. 1986, MNRAS, 221, 839

Nauenberg, M. 1972, ApJ, 175, 417

Nousek, J. A., Takalo, L. O., Schmidt, G. D., et al. 1984, ApJ, 277, 682

Osborne, J. P., Beuermann, K., Charles, P., et al. 1987, ApJ, 315, L123

Osborne, J. P., Bonnet-Bidaud, J.-M., Bowyer, S., et al. 1986, MNRAS, 221, 823

Otazu, X., Ribo, M., Peracaula, M., Paredes, J. M., \& Nunez, J. 2002, MNRAS, 333, 365

Panek, R. J. 1980, ApJ, 241, 1077

Piirola, V., Coyne, G. V., Takalo, S. J., et al. 1994, A\&A, 283, 163

Szatmary, K., Vinko, J., \& Gal, J. 1994, A\&AS, 108, 377

Schwope, A. D., Catalan, M. S., Beuermann, K., et al. 2000, MNRAS, 313,533

Shakhovskoy, N. M., Andronov, I. L., Kolesnikov, S. V., \& Halevin, A. V. 1998, Kinemat. Phys. Celest. Bodies, 14, 468

Soma, M., Hirayama, Th., \& Kinoshita, H. 1988, Celest. Mech., 41, 389

Wu, K., Pongracic, H., Chanmugam, G., \& Shaviv, G. 1996, PASAu, 13,93

Wynn, G. A., \& King, A. R. 1995, MNRAS, 275, 9

Yuan, J., \& Li, Z. 1997, A\&A, 322, 841 